\title{
The In situ Electrochemical Detection of Microbubble Oscillations during Motion through a Channel
}

Received 00th January 20xx, Accepted 00th January 20xx

DOI: $10.1039 / x 0 x x 00000 x$

\author{
Peter R. Birkin ${ }^{\mathrm{a} *}$, Steven Linfield ${ }^{\mathrm{a}}$ and Guy Denuault ${ }^{\mathrm{a}}$
}

\begin{abstract}
Bubble oscillation has many applications from driving local fluid motion to cleaning. However, in order to exploit their action, a full understanding of this motion, particularly in confined spaces (such as crevices etc. which are important in ultrasonic decontamination) is important. To this end, here we show how a Coulter counter can be used to characterize microbubbles produced through the ultrasonication of electrolytes. These microbubbles are shown to exist in relatively high concentrations while bubble activity is driven by ultrasound. Detection of these microbubbles, and their oscillatory behaviour, is achieved via translocation through a cylindrical glass microchannel (GMC). The microbubbles oscillate within the $40 \mu \mathrm{m}$ channel employed and this behaviour is observed to change over the translocation period. This is attributed to the acoustic environment present or changes to the physical conditions in the interior of the chamber compared to the exterior. High-speed imaging confirms the presence of microbubbles as they move or 'skate' across the surface of the structures present before translocating through the channel. The observations are useful as they show that microbubble oscillation occur within small structures, is preceded by surface confined bubbles and could be enhanced through pressure driven flow through a structure.
\end{abstract}

\section{Introduction}

Microbubbles, produced through the action of ultrasound, are associated with the many chemical ${ }^{1-5}$ and physical ${ }^{6-9}$ effects of cavitation. For example, the cleaning ${ }^{10-16}$ of interfaces requires 'active' gas bubbles, driven with an appropriate pressure field, to be present in order for material to be efficiently removed from the surface of an object. Other examples include the use of microbubble excitation for microfluidic applications ${ }^{17}$ or the manipulation/destruction ${ }^{18}$ of contrast agents ${ }^{19}$. Understanding the mechanisms present and the role of an individual bubble is key to the possible exploitation of technologies reliant on microbubble behaviour. However, these bubbles may be transient in nature and the lifetime, location and dynamics of these entities will ultimately dictate their action on the system. As such, the characterization of these bubbles is key to understanding these complex environments. Microbubbles can be detected through the use of high-speed imaging ${ }^{20-22}$ techniques. However, this approach is not suitable for opaque media, requires both high temporal and spatial resolution (e.g. $\mu \mathrm{m}$ and $\mu$ s accuracy) and it is difficult to observe the dynamics of the bubbles as they move through the channel in detail. Hence, there is a considerable need to

\footnotetext{
a. Department of Chemistry, University of Southampton, Southampton, United Kingdom, SO17 1BJ.

Electronic Supplementary Information (ESI) available: Data related to the experimental results presented in the main manuscript is included in the supplementary information along with high-speed imaging sequences. See DOI: $10.1039 / x 0 x x 00000 x$
}

develop new approaches, which would be able to characterize bubbles in situ with a high degree of accuracy. To this end, we have deployed a Coulter counter ${ }^{23-25}$ approach in an effort to both size and monitor the behaviour of microbubbles within a sound field and, critically, as they pass through a channel.

The Coulter counter is a technologically important device that has been used to study a wide variety of particulate materials including polymer spheres 26,27 . At its core is the principle that an electrolyte filled channel (or other restriction) has a characteristic resistance. However, if a material with a different conductivity with respect to the bulk electrolyte enters the channel, the resistance of the structure will change ${ }^{28}$. This perturbation is caused by a displacement principle and the effect the object has on the ionic path through the channel or pore used ${ }^{23,29-31}$. The corresponding change in the resistance is typically monitored by the imposition of a suitable DC bias between two electrodes placed in the interior and exterior spaces with respect to the pore. Changes in the resistance as a particle translocates can then be used, with suitable correction factors, to size the particle accurately.

It would seem that a combination of Coulter counter technology with the ultrasonic generation and excitation of microbubbles is timely, particularly if other key parameters and observations are made. Hence, in this work we discuss the use of Coulter counting to provide a new method of detecting and characterizing microbubbles. These microbubbles, generated by ultrasonication of electrolytes, translocate through a glass microchannel. In so doing so they can be sized from the magnitude of the corresponding current time signal. In 


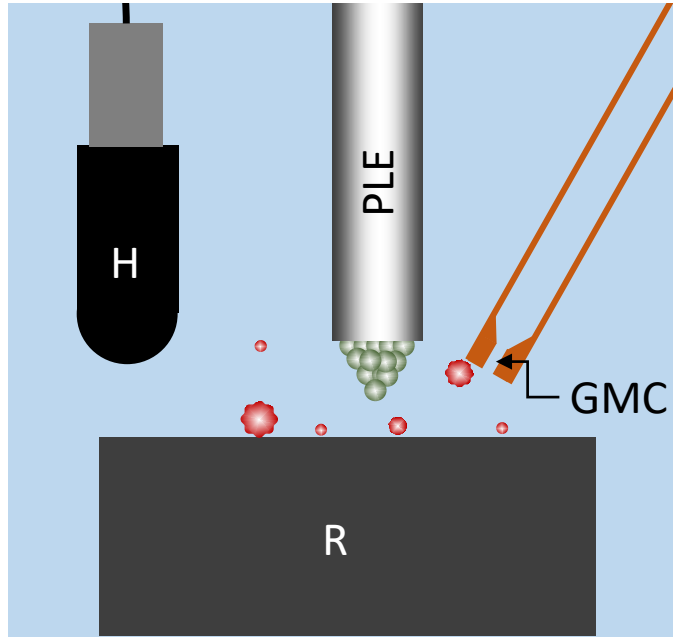

Figure 1. Schematic representation of the generation and detection of microbubbles. Here the sound source (a piston like emitter, PLE) was positioned over a glass reflector $(\mathrm{R})$. The $\mathrm{GMC}$ and the hydrophone $(\mathrm{H})$ were held in place by mechanical positioners (micrometers and stages). ( $\bullet$ ) represents the cluster events at the tip of the PLE, while $(\bullet)$ represents gas bubbles in the bulk or on the surfaces within the system. Note, not to scale.

addition, they are shown to oscillate as they translocate through the microchannel in accordance with the local acoustic environment present. This motion could be useful in cleaning deep within a material/object and could be enhanced by driving flow through a system.

\section{Experimental}

Chemicals and Materials: Hydrochloric acid (35-38\%), hydrogen peroxide $(29.6 \% \mathrm{w} / \mathrm{v})$, potassium chloride $(99.5 \%)$, were purchased from Fischer Scientific and were all used as received. All solutions were made with $18.2 \mathrm{M} \Omega \mathrm{cm}$ purified water from a Suez Select Fusion water system. Copper microwire $(40 \mu \mathrm{m}$ diameter, 99.5\%) was purchased from Advent Research Materials. Soda glass capillary tubes were used as inert substrates for the GMCs employed.

GMC fabrication: The GMCs were fabricated by sealing a copper microwire into soda glass ( $2 \mathrm{~mm}$ OD) capillaries using a small butane flame. These structures were then typically polished with fine emery paper followed by $1 \mu \mathrm{m}$ and $0.3 \mu \mathrm{m}$ alumina on polishing cloth (Buehler) to a mirror like finish. The exposed copper wire (used as a template for the microchannel) was then etched with a mixture of $\mathrm{HCl} / \mathrm{H}_{2} \mathrm{O}_{2}$ (Care) overnight. The final dimensions of the pores were determined by measuring their resistance using conventional cyclic voltammetry in $1 \mathrm{M} \mathrm{KCl}$ and by optical inspection of the structure.

Instrumentation: Electrochemical measurements were performed on a custom-built current follower. Ag wires (Advent Research Materials, $500 \mu \mathrm{m}$ diameter wire) anodized in chloride media, were used as the electrodes in the interior and exterior of the GMC. High-speed camera videos were recorded using a Fastcam-APX RS from Photron. The images were analysed using Photron Fastcam Viewer ver. 3391 software. The geometry and dimensions of the GMC were routinely checked using a $12 \mathrm{X}$ Navitar lens with a variable $0.58 \mathrm{X}-7 \mathrm{X}$ zoom and a $0.67 \mathrm{X}$ adaptor tube was fitted onto a JAl camera unit. A Reson TC4013 hydrophone was used to monitor the acoustic environment within the cell.

Microbubble generation: Ultrasonication of potassium chloride solutions was achieved using a titanium piston-likeemitter (PLE) (3.2 mm diameter) fitted with an insulating ABS thread between the main body and the tip (this minimizes some electrical noise issues). The PLE was powered by a Microson XL2007 ultrasonic cell disruptor from Misonix Inc. The PLE frequency was in the range of $22-23 \mathrm{kHz}$ and the PLE was driven at an output power (derived from the associated instrumentation) reported in the appropriate figure legend. The potassium chloride solutions were held in a custom cell, which housed an optical glass window for the high-speed camera to observe the bubbles. An SQ16 joint at the bottom of the cell allowed a $1 \mathrm{~cm}$ diameter glass reflector to be inserted at variable height. This solid surface was found to improve the reproducibility of microbubble translocation. The tip of the PLE was held $2.5 \mathrm{~mm}$ above the glass cylinder and $5 \mathrm{~mm}$ below the meniscus of the solution. Figure 1 shows a schematic representation of the experimental setup used to generate the microbubbles and detect them electrochemically. Figure S1 shows an image of the relevant experimental setup highlighting the relative components and some of the bubbles events seen. Movie S1 show the bubble activity within this environment. The relative positions of the PLE and GMC were controlled by micropositioners. A static pressure difference was applied across the GMC to draw liquid into the interior of the chamber and to drive bubble translocation. The experimental setup used to generate the static pressure has been described previously ${ }^{32}$.

\section{Results and discussion}

Figure 2 shows typical data recorded during bubble generation and translocation. In the absence of bubble translocation (e.g. at $0 \mathrm{~s}$ and after $2.7 \mathrm{~s}$ ) the current is constant. This current is limited by the dimensions of the pore and the conductivity of

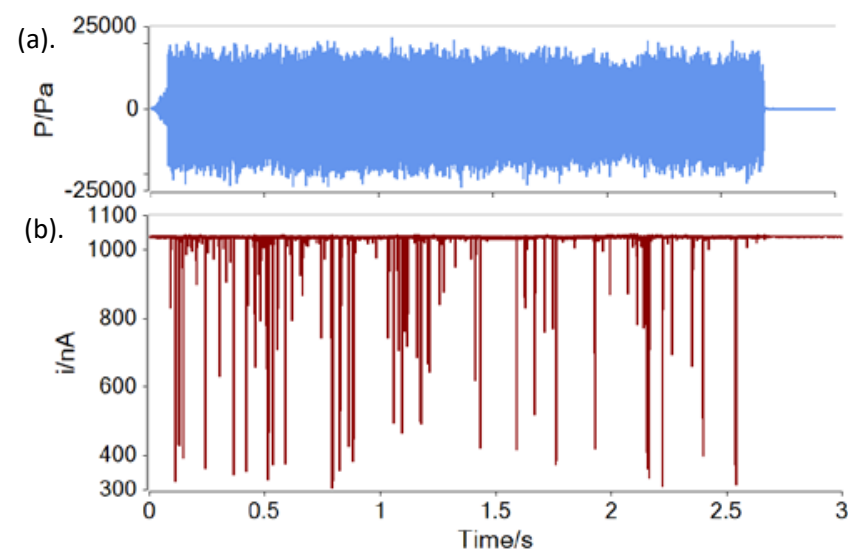

Figure 2. Plots showing the output of the hydrophone $(P,-),(a)$, and ion current $(i$, $-)$, (b), as a function of time for a $40 \mu \mathrm{m}$ diameter pore. The GMC tip was positioned $\sim 1 \mathrm{~mm}$ laterally and level with the PLE (see SI Figure S1) which was operated at 2 $\mathrm{W}_{\mathrm{rms}}$. The aerobic solution contained $10 \mathrm{mM} \mathrm{KCl}$. A potential of $5 \mathrm{~V}$ was applied across the pore. A pressure difference of $76 \mathrm{mBar}$ was applied to draw liquid into the GMC. The solution temperature was $\sim 24{ }^{\circ} \mathrm{C}$. 
the media. In the case here $(10 \mathrm{mM} \mathrm{KCl})$, this base resistance was $4.822 \mathrm{M} \Omega$. Note, increasing the electrolytes concentrations will in turn decrease the base resistance of the pore.

The ultrasonic source (the PLE) was activated at $0 \mathrm{~s}$ and terminated at $\sim 2.7 \mathrm{~s}$. The hydrophone signal indicates exactly when the source was active. In contrast, the Coulter counter signal indicates translocation events. Apart from a short delay in activity, after activating the source, a series of translocation events were detected by the GMC throughout the duration of ultrasonication. The transients in the current time data are attributed to the translocation of microbubbles through the channel. These bubbles occlude the ionic pathway and, as expected, produce an increase in resistance, which is reflected as an accompanied drop in the recorded current. Note that in this experiment, a constant static pressure difference was applied across the GMC. This continually drew liquid into the interior of the GMC structure. The lack of events after terminating (indeed the complete data set showed that there were no events for the complete data capture used after termination of the ultrasonic source) the ultrasonic source indicates that detectable gas bubbles were not present in the absence of the ultrasonic stimuli under these conditions. As is typical for a Coulter counter approach, the size of the current transients (or indeed the depth of the current reduction) is related to the size of the current suppression during translocation. In turn, the variety of magnitudes indicates that the bubbles detected in this manner are part of a polydispersed population. Figure 3(a) shows two examples of the smaller translocations observed for the GMC employed.

Clear oscillations in the current time data can be seen in both cases. These are attributed to the oscillation of the gas bubble diameter in response to the sound field present as it translocated through the microchannel. These size oscillations cause changes in the ion current (here $170 \mathrm{nA}$ peak-to-peak) through the channel and hence can be detected in the data. The amplitude of the current oscillations appears to be greatest as the bubble enters the channel and smallest as it leaves the confines of the GMC. This suggests that the size oscillations diminish as the bubble translocates, presumably as the acoustic amplitude diminishes through the channel or the physical conditions make bubble oscillation more difficult. In addition, imaging shows permanent gas bubbles, which were stationary and attached to the interior of the GMC support structure. These observations suggest that the acoustic field is significant in the channel itself but not within the interior of the GMC support structure. However, the continued activity as the microbubble translocates through the GMC itself is important in that it shows an insight into possible cleaning mechanisms present in such an environment (e.g. channel cleaning). The experiments reported here have direct relevance to channel cleaning in that the measurements made are able to characterise microbubble motion within such a structure. The results suggest that the smaller microbubbles (not slugs) continue to oscillate as the bubble translocates through the $\sim 760 \mu \mathrm{m}$ long channel and, if this motion is responsible for cleaning action (as suggested previously ${ }^{33,34}$ ), indicate that deep cleaning action can be attained through these active bubbles.
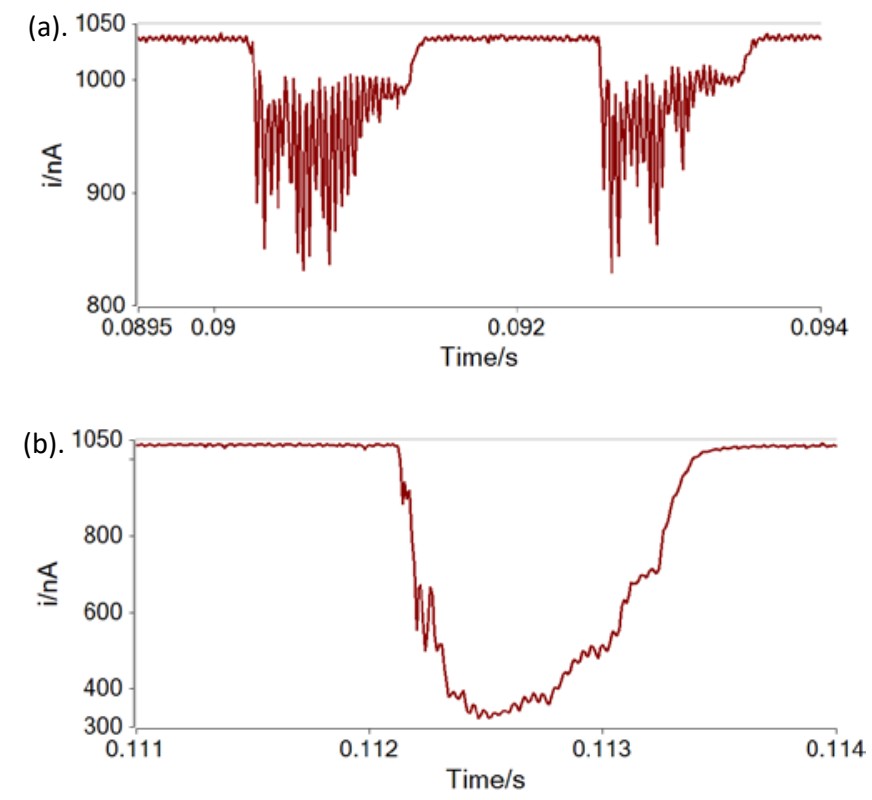

Figure $3(a)$, (b). Plots showing the ion current $(i,-)$ as a function of time for a $40 \mu \mathrm{m}$ diameter pore. The GMC was positioned $\sim 1 \mathrm{~mm}$ laterally and level with the PLE which was operated at $2 \mathrm{~W}_{\text {rms }}$. The aerobic solution contained $10 \mathrm{mM}$ $\mathrm{KCl}$. A potential of $5 \mathrm{~V}$ was applied across the pore. A pressure difference of $75 \mathrm{mBar}$ was applied to draw liquid into the GMC. The solution temperature was $\sim 28^{\circ} \mathrm{C}$.

Presumably, efficient channel cleaning would be enhanced by the application of a static inward pressure gradient. However, surfaces with close crevices (for which the approach adopted here would not be possible) must rely of bubble motion and entrapment without added flow effects. In order to study crevice cleaning, a different experimental protocol would have to be adopted (e.g. a recessed electrode).

The exact upper limit of the particular bubble oscillations shown in figure 3 (a) are difficult to gather from standard Coulter counter theory as this is only applicable for a range of pore to particle ratios. However, in the data set there are even larger translocations present in these experiments. Figure 3(b) shows an example of a large translocation taken from the data shown in figure 2 . If we consider what this current transient represents (assuming the current change with respect to the free channel will be directly related to the void fraction occupied by the gas), figure 3 (b) denotes a void fraction of $\sim 0.68$. This implies that the channel is considerably filled with gas and the bubble is no longer spherical. In this case, the microbubble can be considered as a 'slug' of gas travelling through the GMC. This slug may even fragment as it is pulled through the GMC (see Movie S2 in the supplementary data) under the static pressure applied. Figure 3(a) and (b) suggest that the smaller bubbles are more dynamic in nature and show considerable current time oscillation compared to the larger events. This implies that the 'slug' events (Figure 3(b) for example) are less susceptible to acoustic pressure changes In order to characterize the frequency of the oscillation, an FFT approach was adopted. Figure 4 shows a set of data taken from a GMC in the presence of ultrasonic bubble generation, which has been analysed for the representative frequency components. These components are plotted as a function of 
time. Figure $4(\mathrm{a})$ shows the $\mathrm{DC}$ component (i.e. the ' $\mathrm{OHz}$ frequency component) taken directly from the FFT output. This represents a time-averaged response of the current within the time window employed in the analysis ( $2 \mathrm{~ms}$ ). Using this data, with standard Coulter counter theory, enables the time average size of the gas bubbles to be determined. For example, the translocation events marked with (i), (ii) and (iii), correspond to microbubbles, which are estimated to have a time-averaged diameter of $37.5 \mu \mathrm{m}, 35.0 \mu \mathrm{m}$ and $21.0 \mu \mathrm{m}$ respectively (see SI for calculation details). Figure 4 (b) shows the frequency components present in the current data. This shows

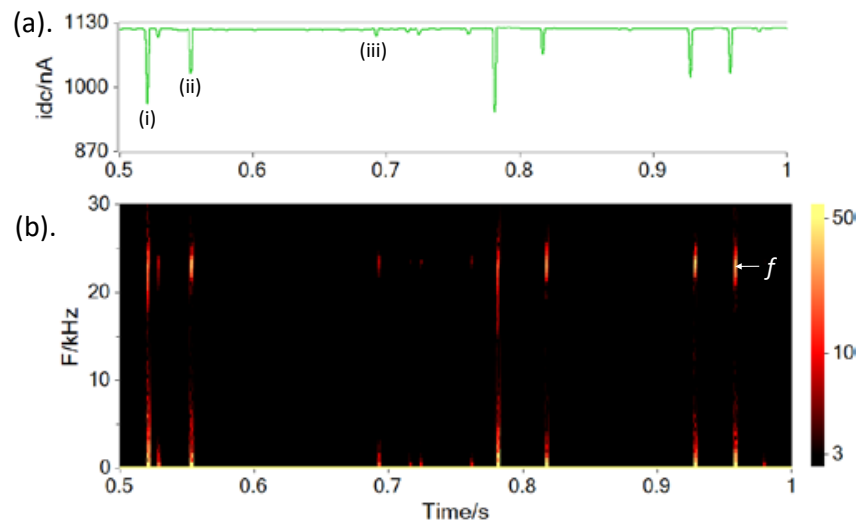

Figure 4. Plots showing (a) the dc ion current (idc, - ) as a function of time for a $40 \mu \mathrm{m}$ diameter pore and (b) the frequency components present in the current time data plotted as a function of time. In (b) the scale bar represents $\mathrm{nA}$. The GMC was positioned $\sim 1 \mathrm{~mm}$ laterally and level with the PLE ( $2 \mathrm{~W}_{\mathrm{rms}}$ ). The aerobic solution contained $10 \mathrm{mM} \mathrm{KCl}$. A potential of $5 \mathrm{~V}$ was applied across the pore. A pressure difference of $75 \mathrm{mBar}$ was applied to draw liquid into the GMC. The solution temperature was $\sim 28^{\circ} \mathrm{C}$. (i, ii, iii) refer to individual transients discussed in the main text. ' $f$ ' denotes the frequency of the PLE.

that the microbubbles are oscillating under these conditions with a frequency of $\sim 23 \mathrm{kHz}$. This corresponds to the frequency of the acoustic source employed and is supported by analysis of the hydrophone data (see figure S3, supplementary information). Interestingly all the translocations, both large and small, appear to have similar frequency components associated with oscillation centred around the acoustic source's frequency apart from low frequency components associated with the transient nature of the translocation event itself. This indicates that the detected population is able to respond to the local acoustic conditions. To investigate how changes in the acoustic environment affect the microbubble behaviour, a series of further experiments were performed where the acoustic conditions were altered (e.g. increased power input). Figure 5 shows an example of the frequency components within the current time data under these conditions. In this case, the power input (as defined by the $W_{\text {rms }}$ reported by the equipment) to the PLE source had been increased in order to change the local acoustic conditions and alter the bubble dynamics in the cell. Figure 5(a) shows a set of bubble transients generated under these conditions. Figure $5(\mathrm{~b})$ shows additional subharmonics in the translocating microbubbles (see annotated frequency components); for some of the microbubbles the subharmonic signals are particularly clear. These subharmonic components are attributed to the local sound field and the presence of acoustic emissions due to cluster ${ }^{35,36}$ dynamics at the PLE tip. Additional evidence for this effect can be found by frequency analysis of the accompanying hydrophone data (see figure S4, supplementary information). The hydrophone data show subharmonic emissions at $2 f / 3$ and $f / 3$ (where $f$ is the ultrasonic source frequency) which are constant through the exposure of the liquid to the acoustic stimulation (apart from a 20-50 ms ring-up 7 region, see figure S4, supplementary information). This is in contrast to the translocation data, which show these subharmonics are only present as bubbles translocate the GMC. This suggests that the microbubbles, and their forced oscillation, are responsible for this signature in the current time data and not noise pickup. Finally, high-speed imaging of the region reveals the origin of these active bubbles. These experiments showed the bubble activity within the system close to the PLE, the microchannel and the glass reflector. The images (and the associated video footage, see $\mathrm{SI}$ ) indicate that this region is extremely complex with bubbles generated and present on the reflector, the PLE tip and the GMC structure. In many cases, the images indicate that oscillating bubbles are present on the surface of the GMC body. These were seen to skate across the surface and some are seen to translocate through the GMC. In some cases, small individual (surface) bubbles can be seen to translocate. Larger bubbles are also drawn through the GMC. In these cases, 'slugs' of bubbles are observed to translocate and may also fragment as they translocate (See SI for examples). These two types of translocation support the data shown in Figure 3. Note, at this resolution, it is difficult to see bulk individual microbubbles moving through the channel. However, this does not preclude their existence, but suggest that this surface mediated route
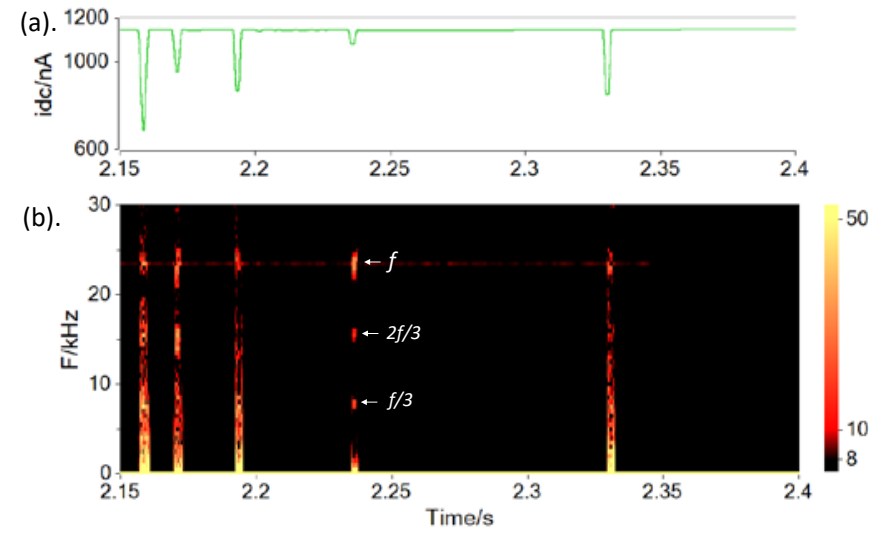

Figure 5. Plots showing (a) the dc ion current (idc, - ) as a function of time for a $40 \mu \mathrm{m}$ diameter pore and (b) the frequency components present in the current time data plotted as a function of time. In (b) the scale bar represents $\mathrm{nA}$. The GMC was positioned $\sim 1 \mathrm{~mm}$ laterally and level with the PLE (18 $\left.\mathrm{W}_{\mathrm{rms}}\right)$. The aerobic solution contained $10 \mathrm{mM} \mathrm{KCl}$. A potential of $5 \mathrm{~V}$ was applied across the pore. A pressure difference of $70 \mathrm{mBar}$ was applied to draw liquid into the GMC. The solution temperature was $\sim 28^{\circ} \mathrm{C}$. The annotation refers to the frequency components and highlights the subharmonics in the current time data. Here $f$ refers to the frequency of the ultrasonic source.

exists in this system. Lastly, the high-speed imaging indicates that the reflector also plays a role with many active gas bubbles skating across its surface. In addition, the close proximity of the PLE to the reflector ensures there is a highly bubble populated region close to the GMC increasing the likelihood of bubble 
detection and characterization (see SI data). This supports the experimental observation that the reflector aids the observation of these events. Lastly, smaller bubbles than those detected and reported here, may be present in this environment. However, in order for those to be analysed, pores with smaller dimensions ${ }^{26}$ compared to the $40 \mu \mathrm{m} \mathrm{GMC}$ would be required.

\section{Conclusions}

Microbubbles, generated by ultrasound, have been successfully detected using a Coulter counter system employing a $40 \mu \mathrm{m}$ diameter channel. Translocation events were only detected when the ultrasonic source was active. Furthermore, the bubbles were found to oscillate as they translocated through the $\sim 760 \mu \mathrm{m}$ long GMC over a 1-2 ms time period. The frequency of bubble oscillation appears to follow the local acoustic conditions associated with the ultrasonic source and the cluster dynamics present. The bubble oscillation is significant and indicates that these active bubbles are likely to play a role in crevice or channel cleaning; a mechanism already associated with ultrasonic systems. The microbubbles detected appear to be present on the surface of the GMC structure and skate over the surface until they are drawn through the channel employed.

\section{Conflicts of interest}

There are no conflicts to declare.

\section{Acknowledgements}

The authors would like to thank the University of Southampton for support for SL and the EPSRC for equipment funding.

\section{Notes and references}

1 K. S. Suslick, The Chemical Effects of Ultrasound, Sci. Am., 1989, 260, 80-86.

2 A. Henglein, Chemical Effects of Continuous and pulsed ultrasound in aqueous solutions., Ultrason. Sonochem., 1995, 2, s115-s121.

3 F. R. Young, Cavitation, Imperial College Press, London, 1999.

$4 \quad$ G. J. Price and E. J. Lenz, The use of dosimeters to measure radical production in aqueous sonochemical systems, Ultrasonics, 1993, 31, 451-456.

5 G. J. Price, Introduction to Sonochemistry, The Royal Society of Chemistry, Cambridge, 1992.

6 P. R. Birkin, D. G. Offin, P. F. Joseph and T. G. Leighton, Cavitation, shock waves and the invasive nature of sonoelectrochemistry, J. Phys. Chem. B, 2005, 109, 1699717005.

7 P. R. Birkin, D. G. Offin, C. J. B. Vian and T. G. Leighton, Multiple observations of cavitation cluster dynamics close to an ultrasonic horn tip, J. Acoust. Soc. Am., 2011, 130, 3379-3388.

P. R. Birkin, D. G. Offin and T. G. Leighton, The study of surface processes under electrochemical control in the presence of inertial cavitation, Wear, 2005, 258, 623-628. A. Philipp and W. Lauterborn, Cavitation erosion by single laser-produced bubbles, J. Fluid Mech., 1998, 361, 75-116. P. R. Birkin, D. G. Offin, C. J. B. Vian and T. G. Leighton, Electrochemical 'Bubble Swarm' Enhancement of Ultrasonic Surface Cleaning, Phys. Chem. Chem. Phys., 2015, 17, 21709-21715.

P. R. Birkin, D. G. Offin, C. J. B. Vian, R. P. Howlin, I. Jonathon, T. J. Secker, R. C. Hervé, P. Stoodley, R. O. C. Oreffo, W. Keevil and T. G. Leighton, Cold water cleaning of brain proteins, biofilm and bone - harnessing an ultrasonically activated stream, Phys. Chem. Chem. Phys., 2015, 17, 20574-20579.

R. P. Howlin, S. Fabbri, D. G. Offin, N. Symonds, K. S. Kiang, R. J. Knee, D. C. Yoganantham, J. S. Webb, P. R. Birkin, T. G. Leighton and P. Stoodley, Removal of Dental Biofilms with an Ultrasonically Activated Water Stream., J. Dent. Res., 2015, 94, 1303-1309.

13 P. R. Birkin, D. G. Offin and T. G. Leighton, An activated fluid stream - new techniques for cold water cleaning, Ultrason. Sonochemistry, 2016, 29, 612-618.

A. A. Busnaina and G. W. Gale, An Experimental Study of Megasonic Cleaning of Silicon Wafers, J. Electrochem. Soc., 1995, 142, 2812-2817.

M. Hauptmann, S. Brems, H. Struyf, P. Mertens, M. Heyns, S. De Gendt and C. Glorieux, Time-resolved monitoring of cavitation activity in megasonic cleaning systems, Rev. Sci. Instrum., 2012, 83, 034904.

M. Hauptmann, H. Struyf, P. Mertens, M. Heyns, S. De Gendt, C. Glorieux and S. Brems, Towards an understanding and control of cavitation activity in $1 \mathrm{MHz}$ ultrasound fields, Ultrason. Sonochem., 2013, 20, 77-88. M. Wiklund, R. Green and M. Ohlin, Acoustofluidics 14: Applications of acoustic streaming in microfluidic devices, Lab Chip, 2012, 12, 2438-2451.

P. P. Kamaev, J. D. Hutcheson, M. L. Wilson and M. R. Prausnitz, Quantification of Optison bubble size and lifetime during sonication dominant role of secondary cavitation bubbles causing acoustic bioeffects, J. Acoust. Soc. Am., 2004, 115, 1818.

19 S. Rossi, G. Waton and M. P. Krafft, Phospholipid-coated gas bubble engineering: key parameters for size and stability control, as determined by an acoustical method., Langmuir, 2010, 26, 1649-55.

O. Lindau and W. Lauterborn, Cinematographic observation of the collapse and rebound of a laserproduced cavitation bubble near a wall, J. Fluid Mech., 2003, 479, 327-348. W. Lauterborn and C. D. Ohl, Cavitation bubble dynamics, Ultrason. Sonochemistry, 1997, 4, 67-75.

Y. Tomita and A. Shima, High speed photographic observations of laser induced cavitation bubbles, Acustica, 1990, 71, 161-171. 
23 R. W. DeBlois and C. P. Bean, Counting and sizing of submicron particles by the resistive pulse technique, Rev. Sci. Instrum., 1970, 41, 909-916.

24 W. H. Coulter, Means for counting particles suspended in a fluid., U.S. Pat., 1953, 9.

25 T. M. Foley, University of Southampton, 2015.

26 L. Luo, S. R. German, W.-J. Lan, D. A. Holden, T. L. Mega and H. S. White, Resistive-Pulse Analysis of Nanoparticles, Annu. Rev. Anal. Chem., 2014, 7, 513-535.

27 W.-J. Lan, D. A. Holden, J. Liu and H. S. White, PressureDriven Nanoparticle Transport across Glass Membranes Containing a Conical-Shaped Nanopore, J. Phys. Chem. C, 2011, 115, 18445-18452.

28 Lord Rayleigh, On the influence of obstacles arranged in rectangular order upon the properties of a medium, Philos. Mag., 1892, 34, 481-502.

29 J. L. Anderson and J. A. Quinn, The relationship between particle size and signal in coulter-type counters, Rev. Sci. Instrum., 1971, 42, 1257-1258.

30 W. R. Smythe, Flow around a spheroid in a circular tube, Phys. Fluids, 1964, 7, 633-638.

31 W. R. Smythe, Flow around a sphere in a circular tube, Phys. Fluids, 1961, 4, 756-759.

32 P. R. Birkin, S. Linfield, G. Denuault, R. Jones, J. Youngs and E. Wain, An analytical differential resistance pulse system relying on a time shift signal analysis - applications in Coulter counting, ACS Sensors, 2019, 4, 2190-2195.

33 D. G. Offin, C. J. B. Vian, P. R. Birkin and T. G. Leighton, An Assessment of Cleaning Mechanisms driven by Power Ultrasound using Electrochemistry and High-Speed Imaging Techniques, Hydroacoustics, 2008, 11, 299-312. D. G. Offin, P. R. Birkin and T. G. Leighton, ARTICLE An electrochemical and high-speed imaging study of micropore decontamination by acoustic bubble entrapment, 2013, 1-3.

35 I. Hansson and K. A. Morch, The dynamics of cavity clusters in ultrasonic (vibratory) cavitation erosion, J. Appl. Phys., 1980, 51, 4651-4658.

36 I. Hansson, V. Kedrinskii and K. A. Morch, On the dynamics of cavity clusters, J. Appl. Phys. D Appl. Phys., 1982, 15, 1725-1734. 